\title{
Carotid Artery and Aortic Stiffness Evaluation in Aortic Stenosis
}

\author{
Sara Hana Weisz, MD, Julien Magne, PhD, Raluca Dulgheru, MD, Pio Caso, MD, Luc A. Piérard, MD, PhD, \\ and Patrizio Lancellotti, MD, PhD, Liège, Belgium; Naples, Italy
}

\begin{abstract}
Background: In aortic stenosis (AS), the combination of risk factors can progressively lead to an increased arterial rigidity, which can be evaluated by the carotid artery and aortic stiffness ( $\beta$ index). The aim of this study was to investigate the relationship between carotid and aortic $\beta$ index, left ventricular (LV) function, plasma brain natriuretic peptide (BNP) level, and symptoms in patients with AS.
\end{abstract}

Methods: Comprehensive echocardiography including Doppler tissue imaging of the mitral annulus was performed in 53 patients with AS (aortic valve area $<1.2 \mathrm{~cm}^{2}$ ) and preserved LV ejection fractions $(\geq 50 \%)$. Carotid $\beta$ index was automatically derived from ultrasound wall tracking of the right carotid artery. The mitral E/e' ratio was used to estimate LV filling pressures.

Results: Carotid $\beta$ index was higher in women than in men and was significantly correlated with age $(P<.0001)$, diastolic arterial pressure $(P=.046)$, pulse pressure $(P=.006)$, and systemic arterial compliance $(P=.001)$. Interestingly, carotid $\beta$ index was significantly correlated with $E / \mathrm{e}^{\prime}$ ratio $(P<.0001)$ and plasma BNP level $(P=.011)$. In multivariate regression analysis, carotid $\beta$ index was an independent predictor of $\mathrm{E} / \mathrm{e}^{\prime}$ ratio $(P<.0001)$ and of BNP level $(P=.02)$. Moreover, carotid $\beta$ index was significantly higher in symptomatic patients $(P=.009)$. Aortic $\beta$ index was significantly correlated with carotid $\beta$ index $(P<.0001)$, E/e' ratio $(P=.004)$, and BNP $(P<.001)$ and was significantly higher in symptomatic patients $(P=.037)$.

Conclusions: In patients with moderate to severe AS and preserved LV ejection fractions, the presence of increased carotid artery and aortic stiffness, assessed using carotid and aortic $\beta$ index, is independently associated with elevated LV filling pressures, BNP level, and symptoms. (J Am Soc Echocardiogr 2014;27:385-92.)

Keywords: Aortic stenosis, Carotid artery stiffness, Aortic stiffness, Symptoms

Calcific aortic stenosis (AS) is the most common valvular disease in Western countries, ${ }^{1}$ and its prevalence increases with population ageing. AS is characterized by an active degenerative process that shares similarities with atherosclerosis. ${ }^{2}$ Currently, AS is no longer considered an isolated aortic valve disease but rather a complex disease in which the central actors are the left ventricle (ability to adapt to the increased afterload), the valve (severity of valvular obstruction), and the vascular system (reduced arterial compliance). ${ }^{3-5}$ As a matter of fact, patients with the same degrees of valvular stenosis can have differing prognoses depending on different degrees of left ventricular (LV) functional compromise or alterations in vascular afterload.

From the Department of Cardiology, GIGA Cardiovascular Sciences, Heart Valve Clinic, University of Liège Hospital, CHU Sart-Tilman, Liège, Belgium (S.H.W., J.M., R.D., L.A.P., P.L.); and Department of Cardiology, Monaldi Hospital, Naples, Italy (S.H.W., P.C.).

This work was supported by the Belgian National Fund for Scientific Research. (F.R.S-FNRS T.0028.14)

Reprint requests: Patrizio Lancellotti, MD, PhD, Department of Cardiology, University Hospital Sart-Tilman, B-4000 Liège, Belgium (E-mail: plancellotti@chu.ulg.ac.be).

0894-7317/ $\$ 36.00$

Copyright 2014 by the American Society of Echocardiography.

http://dx.doi.org/10.1016/j.echo.2013.12.014
In patients with AS, the combination of risk factors (aging process, atherosclerosis, hypertension, etc) can progressively lead to an increased arterial rigidity, ${ }^{6-8}$ which can be evaluated by the assessment of local arterial stiffness at specific sites. ${ }^{9}$ Using twodimensional imaging to measure aortic diameters in patients with AS, it appears that the increased aortic rigidity is independently correlated with LV systolic and diastolic function and brain natriuretic peptide (BNP) levels. ${ }^{8}$ However, measurements of arterial stiffness at different sites of the vascular tree do not seem to be interchangeable, even between the aorta and carotid artery. ${ }^{6,9}$ They are both elastic arteries, but the impacts of different cardiovascular risk factors on their wall properties are not uniform.

So far, in patients with AS, the relationship between carotid arterial stiffness and LV function, BNP, and symptoms has not yet been evaluated. Because of the low sampling rate of B-mode images, the accuracy of two-dimensional imaging for the assessment of carotid stiffness remains limited.' Conversely, ultrasound wall tracking allows more accurate evaluation of vascular diameters, producing precise waveforms of changes during the cardiac cycle. This recent technology can be easily applied to the carotid artery, which is known to be a frequent site affected by the atherosclerotic process. ${ }^{10}$

The aim of this study was to evaluate the impact of carotid artery and aortic stiffness on LV function, BNP release, and clinical status in a series of patients with moderate to severe AS and preserved LV ejection fraction. 


\begin{tabular}{|l|}
\hline \multicolumn{1}{|c|}{ Abbreviations } \\
\hline AS = Aortic stenosis \\
BNP = Brain natriuretic \\
peptide \\
BSA = Body surface area \\
LV = Left ventricular \\
NYHA = New York Heart \\
Association \\
\hline
\end{tabular}

\section{METHODS}

\section{Patient Population}

The present study included a total of 53 patients (mean age, $75 \pm 10$ years; 27 men [51\%]) who underwent comprehensive echocardiography in our Heart Valve Clinic from March 2010 to December 2011 and who fulfilled the following inclusion criteria: moderate to severe AS, defined as an aortic valve area $\leq 1.2 \mathrm{~cm}^{2}$; preserved LV ejection frac tion $(\geq 50 \%$ ); dimension of the ascending aorta $<40 \mathrm{~mm}$ or $\leq 21$ $\mathrm{mm} / \mathrm{m}^{2}$; no significant atherosclerosis of the right carotid artery; and sinus rhythm. Patients with more than mild concomitant mitral valve dysfunction were excluded, as were patients with concomitant aortic insufficiency more than mild in degree. Twenty-six patients had already been included in our previous study of aortic stiffness evaluation. ${ }^{8}$ The following clinical data were collected: age, gender, hypercholesterolemia (total cholesterol $>190 \mathrm{mg} / \mathrm{dL}$ use of lipid-lowering therapy), current smoking, diabetes mellitus, systemic arterial hypertension (blood pressure $\geq 140 / 90 \mathrm{~mm} \mathrm{Hg}$ or use of antihypertensive treatment), and previous evidence of coronary artery disease (presence of $\geq 50 \%$ coronary artery stenosis on angiography, previous revascularization, or previous myocardial infarction). Information regarding current medications was also obtained. The relevant institutional review board approved the protocol, and all patients gave written informed consent.

\section{Measurement of Carotid Artery and Aortic Stiffness}

Subjects were studied after resting supine for $>10 \mathrm{~min}$. Systolic and diastolic blood pressures were measured in the right arm with using an arm-cuff sphygmomanometer at the time of examination. The common right carotid artery was scanned using a Hitachi-Aloka machine (Prosound $\alpha 7$ version 1.1; Aloka, Tokyo, Japan) and a lineararray probe. The change in diameter of the vessel was measured as the difference between the displacement waveforms of the anterior and posterior walls, using the e-tracking technique, with the cursors set manually to track the media-adventitia boundaries in the arterial wall approximately $1 \mathrm{~cm}$ proximal to the carotid sinus. At least 10 sec of consecutive cardiac cycles were recorded for every patient. During offline analysis, carotid artery stiffness ( $\beta$ index) was automatically derived from the average of five cardiac cycles manually selected by the physician and according to the established formula ${ }^{8}$ : $\beta$ index $=\ln (\mathrm{Ps} / \mathrm{Pd}) /[(\mathrm{Ds}-\mathrm{Dd}) / \mathrm{Dd}]$, where $\mathrm{Ps}$ and $\mathrm{Pd}$ are systolic and diastolic blood pressure, and Ds and Dd are the maximal and minimal diameters of the right common carotid artery (Figure 1). Two sets of measurements were performed and averaged for each patient.

The same method was used to record aortic stiffness, $1 \mathrm{~cm}$ above the sinotubular junction by two-dimensionally guided M-mode transthoracic echocardiography in the parasternal long-axis view, as previously described by our group. ${ }^{8}$

\section{Echocardiographic Measurement}

After the assessment of carotid artery stiffness, all patients underwent comprehensive Doppler echocardiographic examinations. Standard echocardiographic views were obtained using second-harmonic imaging. M-mode, two-dimensional, color Doppler, pulse-wave, and
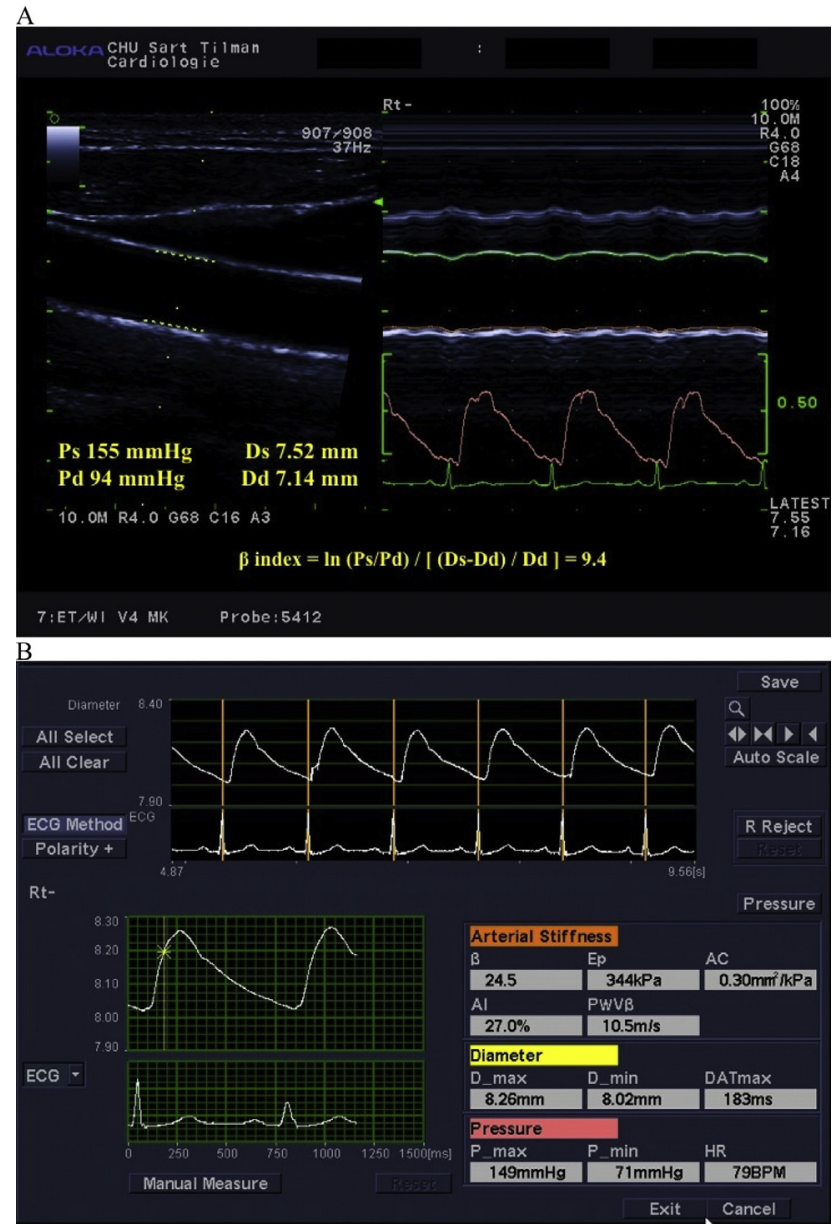

Figure 1 Automatic measurement of carotid $\beta$ index in right carotid artery in a normal subject (A) and a subject with elevated $\beta$ stiffness (B). Dd, Minimal diameter $(\mathrm{mm})$; $D s$, maximal diameter $(\mathrm{mm})$; $P d$, diastolic blood pressure $(\mathrm{mm} \mathrm{Hg}) ; P s$, systolic blood pressure $(\mathrm{mm} \mathrm{Hg})$.

continuous-wave Doppler data were recorded for each patient and were stored in digital format on a dedicated workstation for offline analysis. For each measurement, at least two cardiac cycles were averaged. LV end-diastolic and end-systolic volumes and ejection fraction were measured using the biapical Simpson's disk method. ${ }^{11}$ Continuous-wave Doppler was used to measure the aortic transvalvular maximal velocities; peak and mean gradients were calculated using the simplified Bernoulli equation. Aortic valve area was calculated using the continuity equation (velocity-time integral method) Stroke volume was calculated using the Doppler method as follows:

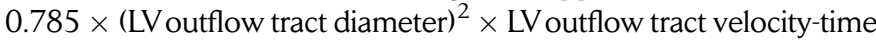
integral. ${ }^{1}$ Peak E-wave and A-wave velocities of mitral inflow were measured using pulsed-wave Doppler. Pulsed-wave tissue Doppler was used to measure systolic $\left(\mathrm{s}^{\prime}\right)$ and early diastolic $\left(\mathrm{e}^{\prime}\right)$ medial mitral annular velocities. The $\mathrm{E} / \mathrm{e}^{\prime}$ ratio was then calculated as an estimate of LV filling pressures. ${ }^{12}$

\section{Global LV Afterload}

To estimate the global LV afterload, valvuloarterial impedance was calculated as the sum of systolic arterial pressure and mean transaortic pressure gradient divided by the stroke volume index. ${ }^{3}$ The ratio between stroke volume index and brachial pulse pressure was used as 
an indirect measure of total systemic arterial compliance and normal values were defined when $>0.6 \mathrm{~mL} / \mathrm{m}^{2} / \mathrm{mm} \mathrm{Hg}^{5}$ Systemic vascular resistance was estimated as the ratio between (mean arterial pressure $\times 80$ ) and cardiac output. ${ }^{5}$

\section{Plasma BNP}

Venous blood samples were obtained before echocardiographic examinations, after resting supine for $>10 \mathrm{~min}$. Chilled ethylenediaminetetraacetic acid tubes were centrifuged immediately at 4,000 $\mathrm{rpm}\left(4^{\circ} \mathrm{C}\right)$ for $15 \mathrm{~min}$. Separated plasma samples were processed by immunofluorescence assay (Biosite; Beckman Coulter, San Diego, CA). Interassay and intra-assay variation was $5 \%$ and $4 \%$, respectively. The assay detection limit was $1 \mathrm{pg} / \mathrm{mL}$.

\section{Symptomatic Status and Risk Score}

Symptomatic status was obtained for each patient with a careful evaluation of patient's history and hospital medical records. Dyspnea was graded according to the New York Heart Association (NYHA) functional class. Patients were classified as symptomatic in the presence of NYHA class $\geq$ II, angina, and/or history of syncope. To differentiate and exclude symptoms potentially related to coronary artery disease, all patients underwent stress echocardiography at least once in the previous 6 months. None of the patients included in the study had wall motion abnormalities at rest or during exercise. A risk score was calculated for all patients according to the following formula: [peak transvalvular velocity $(\mathrm{m} / \mathrm{sec}) \times 2]+[\ln (\mathrm{BNP}) \times 1.5]+1.5$ if female, as previously described by Monin et al. ${ }^{13}$

\section{Statistical Analysis}

Data are expressed as mean \pm SD or as percentages unless otherwise specified. Data on $\beta$ index and BNP were skewed and were thus logarithmically transformed. Log BNP and $\log \beta$ index values were used in correlation and regression analyses as appropriate. Relationships between different parameters were assessed by linear correlation analysis and Pearson's correlation coefficient. To determine the impact of carotid artery stiffness on LV diastolic function, LV filling pressure, BNP plasma level, and symptoms, stepwise linear or logistic multiple regression analyses were performed. Variables with $P$ values $<.10$ on univariate analysis were incorporated into the multiple regression models, with special care to avoid collinearity among a subset of several variables measuring the same phenomenon. Two-sided $P$ values $<.05$ were considered significant. Continuous and nominal variables were compared using Student's $t$ test. Carotid $\beta$ index was compared between symptomatic and asymptomatic patients using a one-way analysis of variance followed by Tukey's test. All statistical analyses were performed using Statistica version 6 (StatSoft Inc, Tulsa, OK).

\section{Reproducibility Analysis}

Interobserver and intraobserver variability for measurement of carotid $\beta$ index was determined from the analysis of 12 randomly selected patients by two independent readers blinded to previous measurements. During offline analysis, each reader was able to select the preferred five cardiac cycles, and carotid $\beta$ index was then automatically derived. Two sets of measurements were performed for each patient and averaged. Absolute difference between repeated measurements was calculated and expressed as the percentage of their mean value for both interobserver and intraobserver results. Moreover, data were compared using intraclass correlation coefficients.
Table 1 Demographic and clinical characteristics $(n=53)$

\begin{tabular}{|cc}
\hline \multicolumn{1}{c}{ Variable } & Value \\
\hline Demographic data & \\
\hline Age $(\mathrm{y})$ & $75 \pm 10$ \\
\hline Men & $27(51 \%)$ \\
\hline Height $(\mathrm{m})$ & $1.67 \pm 0.09$ \\
\hline Weight $(\mathrm{kg})$ & $74 \pm 12$ \\
\hline BSA (m $\left.{ }^{2}\right)$ & $1.82 \pm 0.18$ \\
\hline Body mass index $\left(\mathrm{kg} / \mathrm{m}^{2}\right)$ & $26.5 \pm 3.7$ \\
\hline Clinical data & \\
\hline Systolic arterial pressure $(\mathrm{mm} \mathrm{Hg})$ & $148 \pm 21$ \\
\hline Diastolic arterial pressure $(\mathrm{mm} \mathrm{Hg})$ & $76 \pm 12$ \\
\hline Pulse pressure (mm Hg) & $72 \pm 18$ \\
\hline Heart rate (beats/min) & $69 \pm 12$ \\
\hline BNP (pg/mL) & $101(52-201)$ \\
\hline Symptoms & $17(32 \%)$ \\
\hline Risk factors & \\
\hline Hypercholesterolemia & $41(77 \%)$ \\
\hline Hypertension & $37(69 \%)$ \\
\hline Current smoking & $4(8 \%)$ \\
\hline Previous smoking & $14(26 \%)$ \\
\hline Diabetes mellitus type II & $15(29 \%)$ \\
\hline Coronary artery disease & $20(38 \%)$ \\
\hline Risk score & $15 \pm 3$ \\
\hline Current medications & $14(27 \%)$ \\
\hline ACE inhibitors & $13(24 \%)$ \\
\hline ARBs & $26(49 \%)$ \\
\hline -blockers & $13(24 \%)$ \\
\hline Diuretics & $23(43 \%)$ \\
\hline Nitrates & $10(19 \%)$ \\
\hline
\end{tabular}

$A C E$, Angiotensin-converting enzyme; $A R B$, angiotensin receptor blocker.

Data are expressed as mean $\pm S D$, median (interquartile range), or number (percentage).

\section{RESULTS}

\section{Patient Characteristics}

Table 1 reports demographic and clinical variables, while Table 2 depicts echocardiographic characteristics of the study population. The origin of AS was calcific in 48 patients (91\%) and bicuspid in five (9\%). Seventeen patients (32\%) were symptomatic (dyspnea in 16 , angina in three, syncope in three, combined symptoms in five). Carotid $\beta$ index distribution is reported in Figure 2.

\section{Carotid Artery Stiffness, Clinical Data, Global Afterload, and LV Function}

Univariate correlations between carotid $\log \beta$ index and clinical and echocardiographic variables are listed in Table 3. Carotid $\beta$ index was higher in women than in men $(14.1 \pm 5.0$ vs $11.0 \pm 3.9, P=$ $.02)$ and was significantly correlated with age $(r=0.52, P<.0001)$, body surface area (BSA) $(r=-0.32, P=.02)$, diastolic arterial pressure $(r=-0.28, P=.046)$, pulse pressure $(r=0.38, P=.006)$, and systemic arterial compliance $(r=-0.44, P=.001)$. Dividing patients according to gender, we found significant differences, with higher values in men compared to women for BSA $(P<.0001)$, LV diastolic and systolic volumes $(P<.0001$ for both), indexed diastolic and systolic volumes $(P=.004$ and $P=.007$, respectively) and LV mass 
Table 2 Echocardiographic data $(n=53)$

\begin{tabular}{|c|c|}
\hline Variable & Value \\
\hline \multicolumn{2}{|l|}{ LV geometry } \\
\hline LVEDV (mL) & $76 \pm 22$ \\
\hline LVESV (mL) & $27 \pm 10$ \\
\hline LVEDV index $\left(\mathrm{mL} / \mathrm{m}^{2}\right)$ & $42 \pm 11$ \\
\hline LVESV index $\left(\mathrm{mL} / \mathrm{m}^{2}\right)$ & $15 \pm 5$ \\
\hline LV mass $(\mathrm{g})$ & $196 \pm 57$ \\
\hline LV mass index $\left(\mathrm{g} / \mathrm{m}^{2}\right)$ & $108 \pm 29$ \\
\hline \multicolumn{2}{|l|}{ LV systolic function } \\
\hline Stroke volume (mL) & $80 \pm 19$ \\
\hline Cardiac output (L/min) & $5.4 \pm 1.3$ \\
\hline Ejection fraction (\%) & $65 \pm 7$ \\
\hline $\mathrm{s}^{\prime}$-wave velocity $(\mathrm{cm} / \mathrm{sec})$ & $6.1 \pm 1.5$ \\
\hline \multicolumn{2}{|l|}{ LV diastolic function } \\
\hline E-wave velocity $(\mathrm{cm} / \mathrm{sec})$ & $82 \pm 24$ \\
\hline E/A ratio & $0.9 \pm 0.4$ \\
\hline $\mathrm{e}^{\prime}$-wave velocity $(\mathrm{cm} / \mathrm{sec})$ & $5.5 \pm 1.5$ \\
\hline $\mathrm{a}^{\prime}$-wave velocity $(\mathrm{cm} / \mathrm{sec})$ & $8.0 \pm 2.0$ \\
\hline $\mathrm{E} / \mathrm{e}^{\prime}$ ratio & $16.0 \pm 6.2$ \\
\hline \multicolumn{2}{|l|}{ AS severity } \\
\hline Aortic valve area $\left(\mathrm{cm}^{2}\right)$ & $0.87 \pm 0.24$ \\
\hline Aortic valve area index $\left(\mathrm{cm}^{2} / \mathrm{m}^{2}\right)$ & $0.48 \pm 0.13$ \\
\hline Peak aortic velocity $(\mathrm{m} / \mathrm{sec})$ & $3.9 \pm 0.7$ \\
\hline Mean pressure gradient $(\mathrm{mm} \mathrm{Hg})$ & $41 \pm 16$ \\
\hline \multicolumn{2}{|l|}{ LV global afterload } \\
\hline Energy loss index $\left(\mathrm{cm}^{2} / \mathrm{m}^{2}\right)$ & $0.51 \pm 0.15$ \\
\hline Systemic arterial compliance $\left(\mathrm{mL} / \mathrm{m}^{2} / \mathrm{mm} \mathrm{Hg}\right)$ & $0.65 \pm 0.21$ \\
\hline Systemic vascular resistance (dynes $\cdot \mathrm{s} \cdot \mathrm{cm}^{-5}$ ) & $1,560 \pm 396$ \\
\hline Valvuloarterial impedance $\left(\mathrm{mm} \mathrm{Hg} / \mathrm{mL} / \mathrm{m}^{2}\right)$ & $4.5 \pm 1.0$ \\
\hline \multicolumn{2}{|l|}{ LA dimensions } \\
\hline LA area $\left(\mathrm{cm}^{2}\right)$ & $20.1 \pm 4.5$ \\
\hline $\mathrm{LA}$ area index $\left(\mathrm{cm}^{2} / \mathrm{m}^{2}\right)$ & $11.0 \pm 2.4$ \\
\hline Transtricuspid pressure gradient $(\mathrm{mm} \mathrm{Hg})$ & $25 \pm 9$ \\
\hline
\end{tabular}

LA, Left atrial; LVEDV, LV end-diastolic volume; LVESV, LV endsystolic volume.

Data are expressed as mean \pm SD.

$(P=.02)$. Conversely, no significant gender difference was detected between systolic and diastolic blood pressure $(P=.68$ and $P=.39$, respectively) or indexed LV mass $(P=.31)$. No significant association was found between carotid $\beta$ index and cardiovascular risk factors, coronary artery disease, medications, or absolute or indexed aorta diameter $(P=\mathrm{NS})$. Carotid $\beta$ index was not significantly different in patients with hypertension or diabetes. Moreover, it was not significantly correlated with parameters of AS severity or with valvuloarterial impedance $(P=\mathrm{NS})$, but it was significantly correlated with $s^{\prime}$-wave velocity $(r=-0.35, P=.011)$. There was a trend for significant correlation between transtricuspid pressure gradient and carotid $\beta$ index $(r=0.29, P=.057)$.

\section{Carotid Stiffness and E/e' Ratio}

$\mathrm{E} / \mathrm{e}^{\prime}$ ratio was significantly correlated with age $(r=0.43, P=.002)$, LV end-diastolic volume $(r=-0.29, P=.039)$, left atrial area index $(r=0.29, P=.044), s^{\prime}$-wave velocity $(r=-0.41, P=.003)$, risk score $(r=0.45, P=.002)$ and carotid $\beta$ index $(r=0.41, P=.003)$ (Figure 3$)$. There were no other significant correlations between $\mathrm{E} / \mathrm{e}^{\prime}$ ratio and echocardiographic parameters such as AS severity or valvuloarterial

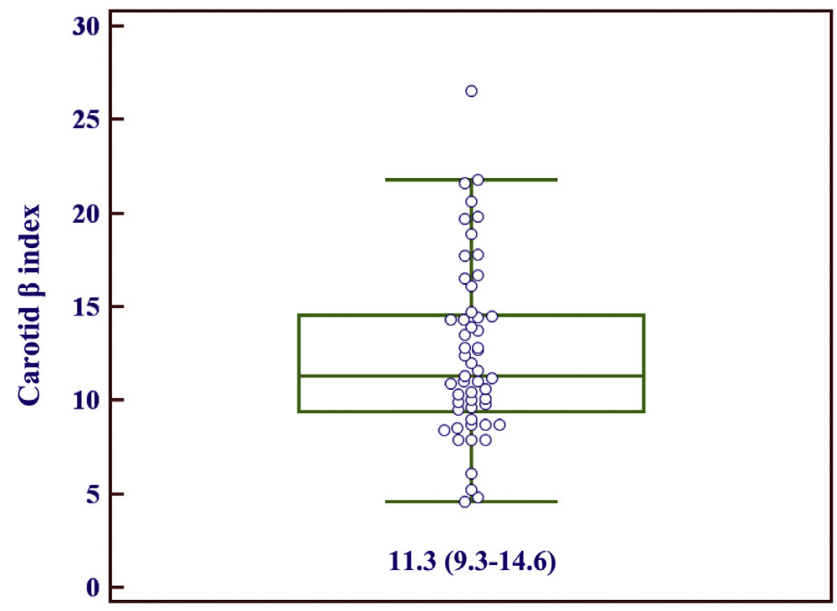

Figure 2 Box plot of carotid artery stiffness distribution in the whole cohort. Values are expressed as median (interquartile range).

impedance. In multiple linear regression analysis, after adjustment for cofactors, left atrial area index $(\beta=1.08 \pm 0.33, P=.002)$ and carotid $\beta$ index $(\beta=20.45 \pm 4.36, P<.0001)$ emerged as independently associated with $\mathrm{E} / \mathrm{e}^{\prime}$ ratio (model $r^{2}=0.45, P<.0001$ ).

\section{Carotid Stiffness and BNP Release}

Log BNP was significantly correlated with $s^{\prime}$-wave velocity $(r=-0.32$, $P=.031)$, peak aortic velocity $(r=0.36, P=.012)$, carotid $\beta$ index $(r=$ $0.37, P=.011)$ (Figure 4$)$, left atrial area $(r=0.39, P=.008)$, age $\geq 77$ years $(P=.003)$, and $\mathrm{E} / \mathrm{e}^{\prime}$ ratio $(r=0.54, P<.0001)$. In multiple linear regression analysis, after adjustment for cofactors, peak aortic velocity $(\beta=0.16 \pm 0.07, P=.027)$, carotid $\beta$ index $(\beta=0.88 \pm 0.36, P=$ $.02)$, and left atrial area $(\beta=0.038 \pm 0.01, P=.007)$ emerged as independently associated with BNP (model $r^{2}=0.50, P<.0001$ ).

\section{Carotid Stiffness and Symptoms}

At the time of echocardiographic evaluation, 17 patients (32\%) had symptoms related to AS. They were significantly older compared with asymptomatic patients $(80 \pm 8$ vs $72 \pm 10$ years, $P=.015)$. Carotid $\beta$ index was significantly higher in patients with symptoms compared to asymptomatic patients $(P=.009$; Figure 5A) and also in patients in NYHA class III compared with those in NYHA class I $(P=.011$; Figure 5B). In addition, according to the median of risk score distribution, carotid $\beta$ index was significantly higher in patients with scores $\geq 15.3$ compared with scores $<15.3(14.2 \pm 5.2$ vs $11.0 \pm 3.8, P=.029$ ). After adjustment for age, sex, and measures of AS severity, carotid $\beta$ index remained significantly associated with symptoms (odds ratio, 1.18; 95\% confidence interval, 1.03-1.36; $P=.017)$

\section{Aortic Stiffness, Carotid Stiffness, E/e', BNP Release and Symptoms}

Aortic $\beta$ index was positively and significantly correlated with carotid $\beta$ index $(r=0.55, P<.001$; Figure 6$)$. Moreover, it was significantly correlated with age $(r=0.37, P=.017)$, pulse pressure $(r=0.38$, $P=.015)$, and systemic arterial compliance $(r=-0.43, P=.005)$. Aortic $\beta$ index was also significantly correlated with $\mathrm{E} / \mathrm{e}^{\prime}$ ratio $(r=$ $0.44, P=.004)$, and BNP $(r=0.54, P<.001)$ and was significantly higher in symptomatic patients $(P=.037)$. There were no significant 
Table 3 Correlations with carotid arterial stiffness

\begin{tabular}{|c|c|c|}
\hline Variable & $r$ & $P$ \\
\hline \multicolumn{3}{|l|}{ Demographic and clinical data } \\
\hline Age & 0.52 & $<.0001$ \\
\hline Gender & 0.25 & .07 \\
\hline Height & -0.35 & .010 \\
\hline BSA & -0.32 & .020 \\
\hline Body mass index & 0.01 & .934 \\
\hline Systolic arterial pressure & 0.15 & .275 \\
\hline Diastolic arterial pressure & -0.28 & .046 \\
\hline Pulse pressure & 0.38 & .006 \\
\hline Heart rate & 0.01 & .934 \\
\hline Log BNP & 0.37 & .011 \\
\hline Risk score & 0.36 & .012 \\
\hline \multicolumn{3}{|l|}{ LV geometry } \\
\hline LVEDV & -0.46 & $<.0001$ \\
\hline LVESV & -0.50 & $<.0001$ \\
\hline LVEDV index & -0.37 & .006 \\
\hline LVESV index & -0.43 & .001 \\
\hline LV mass & -0.32 & .022 \\
\hline LV mass index & -0.19 & .182 \\
\hline \multicolumn{3}{|l|}{ LV systolic function } \\
\hline Stroke volume & -0.23 & .093 \\
\hline Cardiac output & -0.13 & .371 \\
\hline Ejection fraction & 0.23 & .099 \\
\hline $\mathrm{s}^{\prime}$-wave velocity & -0.35 & .011 \\
\hline \multicolumn{3}{|l|}{ LV diastolic function } \\
\hline E-wave velocity & 0.05 & .704 \\
\hline E/A ratio & -0.08 & .598 \\
\hline $\mathrm{e}^{\prime}$-wave velocity & -0.54 & $<.0001$ \\
\hline$a^{\prime}$-wave velocity & -0.14 & .329 \\
\hline E/e' ratio & 0.41 & .003 \\
\hline \multicolumn{3}{|l|}{ AS severity } \\
\hline Aortic valve area & -0.20 & .142 \\
\hline Aortic valve area index & -0.09 & .530 \\
\hline Peak aortic velocity & 0.01 & .920 \\
\hline Mean pressure gradient & 0.02 & .879 \\
\hline \multicolumn{3}{|l|}{ LV global afterload } \\
\hline Aortic $\beta$ index & 0.55 & $<.001$ \\
\hline Energy loss index & -0.11 & .494 \\
\hline Systemic arterial compliance & -0.44 & .001 \\
\hline Systemic vascular resistance & 0.06 & .668 \\
\hline Valvuloarterial impedance & 0.15 & .269 \\
\hline \multicolumn{3}{|l|}{ Left atrium } \\
\hline LA area & -0.06 & .654 \\
\hline LA area index & 0.10 & .495 \\
\hline Transtricuspid pressure gradient & 0.29 & .057 \\
\hline
\end{tabular}

$L A$, Left atrial; LVEDV, LV end-diastolic volume; LVESV, LV endsystolic volume.

correlations between aortic stiffness and parameters of AS severity or valvuloarterial impedance $(P=\mathrm{NS})$. Aortic $\beta$ index was not significantly different in patients with or without hypertension and diabetes $(P=\mathrm{NS})$.

\section{Interobserver and Intraobserver Variability}

Absolute differences between interobserver and intraobserver measurements of carotid $\beta$ index showed low variability, with $9 \pm 7 \%$ and $6 \pm 4 \%$ differences, respectively. Intraclass correlation coeffi-

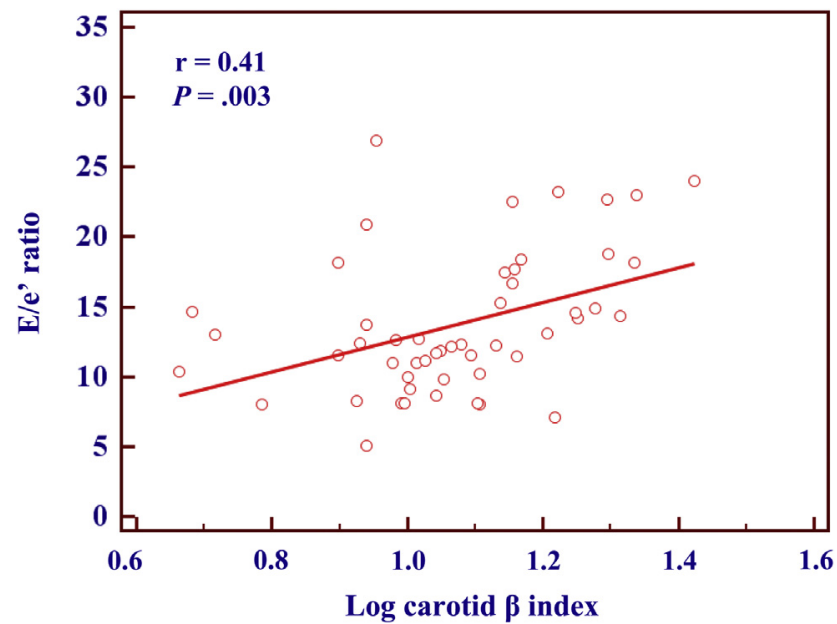

Figure 3 Relationship between estimated LV filling pressures and carotid arterial stiffness.

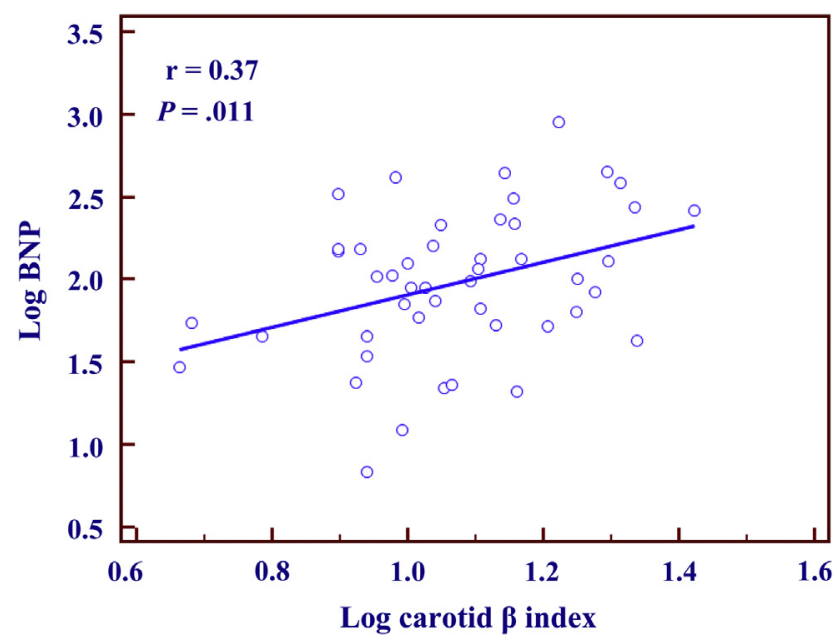

Figure 4 Relationship between BNP and carotid arterial stiffness.

cients demonstrated good interobserver $(0.95 ; 95 \%$ confidence interval, $0.84-0.99)$ and intraobserver $(0.97 ; 95 \%$ confidence interval, 0.91-0.99) agreement.

\section{DISCUSSION}

The main results of the present study are that in patients with moderate to severe AS and preserved LV ejection fractions, increases in carotid $\beta$ index are independently associated with (1) age and female gender; (2) increased $\mathrm{E} / \mathrm{e}^{\prime}$ ratio, an estimate of LV filling pressure, (3) higher BNP level; and (4) symptoms. Moreover, aortic stiffness was also significantly associated with LV filling pressure, BNP level, and symptoms.

\section{Carotid Artery Stiffness and Aortic Stiffness}

Arterial tree ageing determines a progressive modification of arterial wall properties. Several studies have focused their attention on this phenomenon and found a nonuniform process in proximal compared with distal arteries. $6,7,14,15$ Proximal elastic arteries, such as the aorta and common carotid artery, dilate, increase wall 


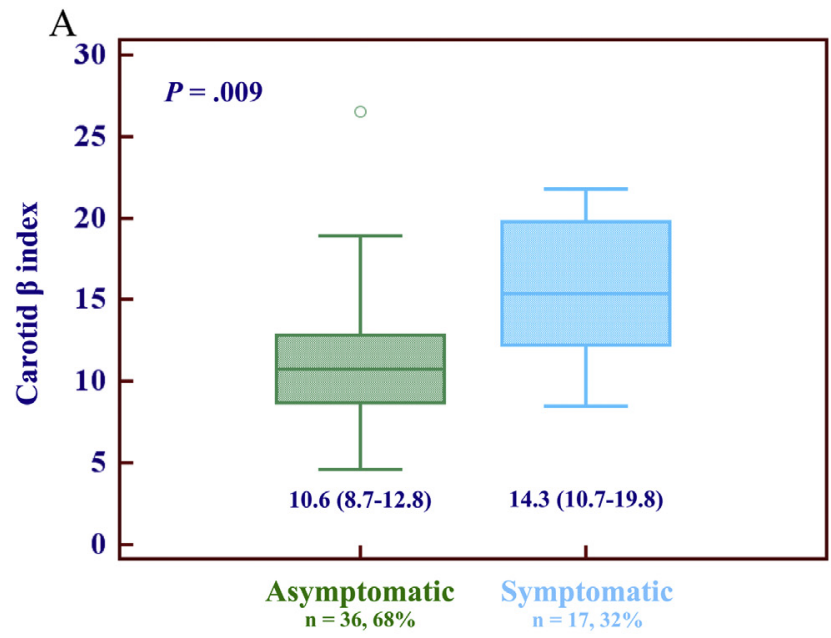

\section{$\mathrm{B}$}

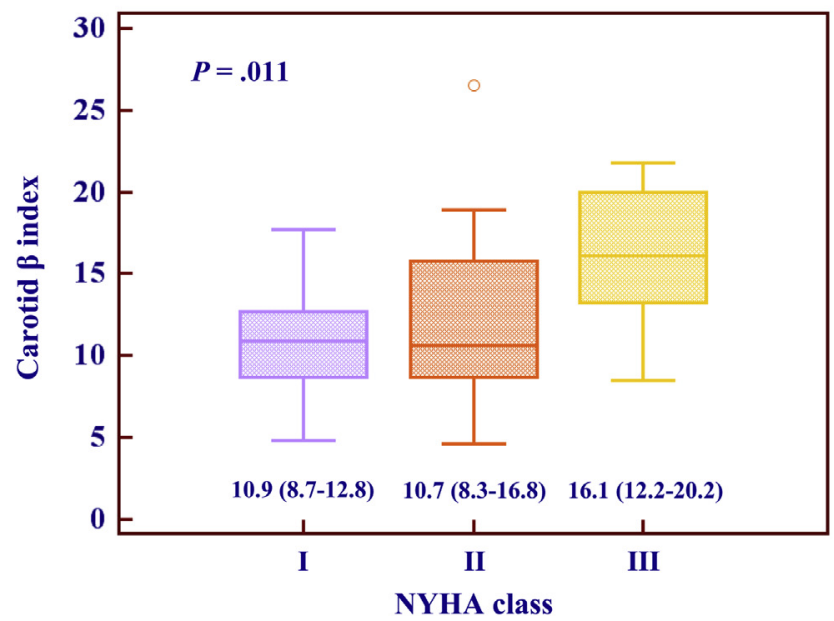

Figure 5 Comparison of carotid arterial stiffness according to symptomatic status (A) and NYHA functional class (B). Values are expressed as median (interquartile range).

thickness, and stiffen. Hypotheses suggest modification in wall composition with degeneration of elastic fibers and augmentation of collagen and mucopolysaccharides. ${ }^{6}$ These structural changes correspond to atherosclerosis and can be recorded in clinical examination as increased pulse pressure, pulse wave velocity, or $\beta$ index. On the other hand, distal muscular medium-sized arteries, such as the brachial artery, have different properties, and their stiffness does not seem to increase with age. ${ }^{6}$ Aorta and carotid wall properties are not always identical in the same subject, although both are elastic arteries. Actually, they both increase with age, especially in women after menopause, but can differ in patients with various cardiovascular risk factors due to nonuniform impact of these factors (especially hypertension and diabetes) on atherosclerosis progression at different sites of the arterial tree. ${ }^{16,17}$

In our population, the impact of carotid and aortic stiffness was similar. Both were associated with impaired diastolic function, increased plasma BNP, and symptoms, and these results are in line with previously published data by our group in patients with severe AS. ${ }^{8}$ All three aspects are usually found in patients with a more advanced stage of disease and carry important prognostic information. For this reason, our results underline once more the importance of studying vascular afterload in these patients.

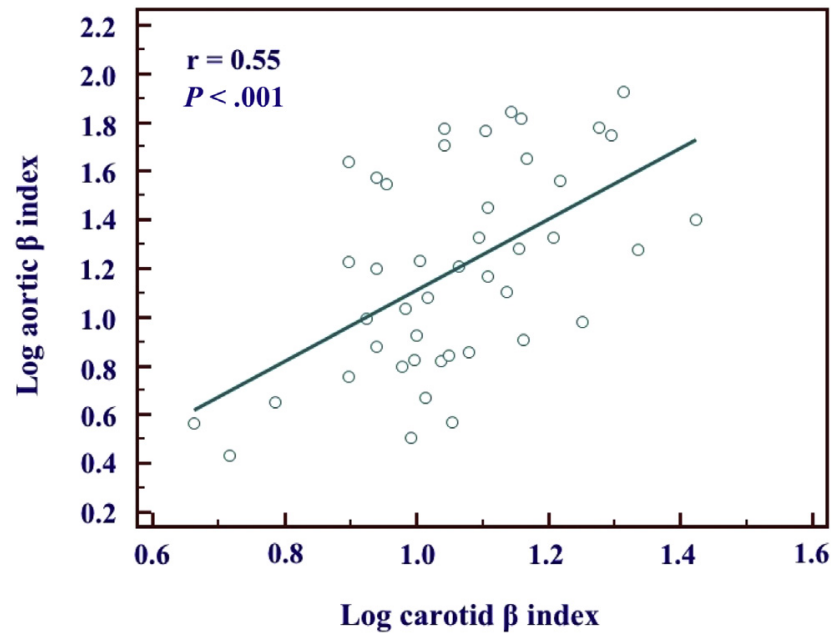

Figure 6 Relationship between aortic and carotid arterial stiffness.

\section{Cofactors Associated with Carotid Arterial Stiffness}

In our study, we confirmed previous findings about gender and age differences in carotid stiffness, with significantly higher carotid stiffness in women and significant positive correlations with age and pulse pressure. An age-related increase in elastic arterial stiffness determines a progressive increment of systolic arterial pressure and pulse pressure and therefore increased LV afterload with LV hypertrophy and dysfunction. ${ }^{5}$

As reported in Table 3, carotid $\beta$ index was higher in patients with lower BSAs and LV volumes and mass. This apparently singular finding can be explained by gender differences. Actually, BSA and LV volumes and mass differed significantly between men and women and were higher in men. Interestingly, there were no significant differences in systolic and diastolic blood pressures between men and women that may explain differences in carotid stiffness calculation, and if LV mass was indexed, the difference between genders was no longer significant. Of note, gender difference in carotid stiffness is similar to the difference reported with aortic stiffness in $\mathrm{AS}^{8}$ Hence, carotid arterial stiffness provides similar information about the impact of aging and gender on large arteries' rigidity in AS.

On the other hand, lower carotid arterial stiffness was correlated with higher systemic arterial compliance, but not with higher global LV afterload as evaluated by valvuloarterial impedance. This is somewhat related to the fact that both carotid arterial stiffness and systemic arterial compliance reflect the load imposed by the vascular system itself on the heart, rather than be markers of ventricular-vascular coupling. In fact, parameters such as energy loss index, valvuloarterial impedance, systemic arterial compliance, systemic vascular resistance, and pulse pressure are not necessarily interchangeable, but they represent various components of global LV afterload with different effects on LV function or disease progression.

In our population, we did not find any relationship between parameters of AS severity (peak aortic jet velocity, transvalvular mean pressure gradient, or aortic valve area) and carotid arterial stiffness. AS has been associated with various degrees of vascular atherosclerosis. Hence, even if they share similar risk factors and histopathologic features, they are different disease. ${ }^{15}$ Antonini-Canterin et al. ${ }^{18}$ also confirmed the absence of correlation between carotid arterial stiffness and classical parameters of AS severity but reported a positive correlation with stroke work loss, an index representing the amount of energy the LV dissipates as heat due to outflow obstruction. ${ }^{19}$ 


\section{Carotid Arterial Stiffness and LV Function in AS}

Long-standing AS with chronic pressure overload leads to compensatory remodeling and reduced end-systolic wall stress. Compensated pathologic hypertrophy reduces wall stress but progressively determines impairment of compliance with elevated LV filling pressures. Elevated LV diastolic pressure associated with reduced arterial diastolic blood pressure limits the coronary flow reserve and leads to subendocardial ischemia, even in the absence of significant coronary artery disease. ${ }^{20}$ Hence, longitudinal function, governed by the subendocardial fibers, is the first to be altered, while LV ejection fraction, depending more on midwall myocardial fibers, is maintained within the normal range until the compensatory mechanisms are exhausted. 8,20 Increased arterial stiffness and reduced systemic arterial compliance have been related to impaired LV diastolic and systolic function in various cardiac conditions. ${ }^{5,14,21}$ In the present study, we have examined, for the first time, the relationship between carotid arterial stiffness and LV function. An increase in carotid artery stiffness, independent of AS severity, LV ejection fraction, or the degree of LV hypertrophy, was directly associated with a significant decrease in LV diastolic performance and an increase in LV filling pressure.

The left ventricles of patients with AS and augmented arterial stiffness face a double afterload, represented by valvular obstruction and vascular reduced compliance. For this reason, LV diastolic dysfunction can occur earlier compared with patients without impaired arterial stiffness. The evidence of LV diastolic dysfunction is not just an echocardiographic finding but also has an important prognostic role, because increased LV filling pressure is independently associated with worse outcomes, such as as onset of symptoms, cardiac-related death, and aortic valve replacement. ${ }^{12}$

In all patients with AS, at least the simple measurement of arterial blood pressure should be effectuated at every echocardiographic examination, because even the concomitant presence of systemic hypertension may cause an underestimation of AS severity. Moreover, the evaluation of carotid artery stiffness, as a more specific marker of increased vascular afterload, can represent a useful additional diagnostic tool.

\section{Carotid Arterial Stiffness, BNP and Symptomatic Status in AS}

BNP plasma level carries important clinical and prognostic information in patients with AS. Higher values of BNP are found in patients with AS compared with controls and are related to higher extent of LV hypertrophy and to AS severity. Moreover, BNP is significantly higher in symptomatic patients with differences according to NYHA class and, like LV filling pressure, has the capacity to predict reduced cardiac event-free survival in both symptomatic and asymptomatic patients. ${ }^{12,22}$ In the present study, we found a significant positive relationship between carotid artery stiffness and plasma BNP, even after adjustment for cofactors, suggesting that patients with higher degree of vascular stiffness are probably at a more advanced stage of the disease. Of note, discordant results have been found when considering the relationship between BNP and other markers of arterial stiffness such as carotid-radial or femoral pulse wave velocity. ${ }^{23}$ This suggests that pulse wave velocity and carotid $\beta$ index do not necessarily provide similar information.

In this study, we found a significant positive relationship between carotid artery stiffness and symptoms, with higher values in NYHA class III compared with class I patients. In AS, symptom onset is a very important event, because prognosis dramatically falls and rapid surgery is required. In clinical practice, the assessment of symptomatic status is not always easy, because of the possible underestimation by patients or self-reduction of their daily activities. In our study population, almost all symptomatic patients had dyspnea, which is mainly related to LV diastolic function and filling pressure.

\section{Study Limitations}

The main limitation of our study was the relative heterogeneity of the studied cohort, including both asymptomatic and symptomatic patients with a wide range of AS severity. Nonetheless, this inclusion criterion is representative of the wide spectrum of AS disease as commonly seen in outpatient clinics. Although some differences may exist, brachial blood pressure was used as a substitute for carotid blood pressure. However, because most of our patients were elderly, the variation in pulse pressure between central and peripheral arteries is attenuated. Nevertheless, several epidemiologic studies have used similar substitutions, and brachial blood pressure remains the standard measure in clinical practice. ${ }^{24,25}$ The sample size of the present study is relatively small and may lead to type II error. This could explain, at least in part, the lack of a relationship between carotid artery stiffness and coronary artery disease. In addition, this could also explain, as well as the inclusion of patients with moderate AS, the absence of an overt significant relationship among LV function, symptoms, and AS severity parameters. Of note, although they were significantly correlated ( $r=0.30, P=.028)$, a discrepancy between the mean stroke volume measurements obtained by the two-dimensional technique and the Doppler technique was observed in the present study. Finally, in our study, although we excluded patients with dilated ascending aortas $\left(>21 \mathrm{~mm} / \mathrm{m}^{2}\right)$, we could not completely exclude different aortic wall properties in these patients.

\section{CONCLUSIONS}

In patients with moderate to severe AS and preserved LV ejection fractions, impaired LV function, elevated plasma BNP, and symptoms are correlated with carotid arterial and aortic stiffness. Because these consequences of AS are well recognized as predictors of poor outcomes, the evaluation of carotid artery and aortic stiffness may be very useful in the management of these patients and might potentially identify patients at more advanced stages of the disease. Nevertheless, further studies are needed to determine whether these indices can also carry important prognostic information and be useful for risk stratification in AS.

\section{ACKNOWLEDGMENT}

We thank Carmine Celentano for excellent technical assistance.

\section{REFERENCES}

1. Baumgartner H, Hung J, Bermejo J, Chambers JB, Evangelista A, Griffin BP, et al., American Society of Echocardiography; European Association of Echocardiography. Echocardiographic assessment of valve stenosis: EAE/ASE recommendations for clinical practice. Eur J Echocardiogr 2009; 10:1-25.

2. Freeman RV, Otto CM. Spectrum of calcific aortic valve disease: pathogenesis, disease progression, and treatment strategies. Circulation 2005; 111:3316-26.

3. Hachicha Z, Dumesnil JG, Pibarot P. Usefulness of the valvuloarterial impedance to predict adverse outcome in asymptomatic aortic stenosis J Am Coll Cardiol 2009;54:1003-11. 
4. Lancellotti P, Donal E, Magne J, Moonen M, O'Connor K, Daubert JC, et al. Risk stratification in asymptomatic moderate to severe aortic stenosis: the importance of the valvular, arterial and ventricular interplay. Heart 2010;96:1364-71.

5. Briand M, Dumesnil JG, Kadem L, Tongue AG, Rieu R, Garcia D, et al. Reduced systemic arterial compliance impacts significantly on left ventricular afterload and function in aortic stenosis: implications for diagnosis and treatment. J Am Coll Cardiol 2005;46:291-8.

6. Van der Heijden-Spek JJ, Staessen JA, Fagard RH, Hoeks AP, Boudier HA, van Bortel LM. Effect of age on brachial artery wall properties differs from the aorta and is gender dependent: a population study. Hypertension 2000;35:637-42.

7. Boutouyrie P, Laurent S, Benetos A, Girerd XI, Hoeks AP, Safar ME Opposing effects of ageing on distal and proximal large arteries in hypertensives. J Hypertens Suppl 1992; 10:S87-91.

8. Roșca M, Magne J, Călin A, Popescu BA, Piérard LA, Lancellotti P. Impact of aortic stiffness on left ventricular function and B-type natriuretic peptide release in severe aortic stenosis. Eur J Echocardiogr 2011;12:850-6.

9. Laurent S, Cockcroft J, Van Bortel L, Boutouyrie P, Giannattasio C, Hayoz D, et al., European Network for Non-Invasive Investigation of Large Arteries. Expert consensus document on arterial stiffness: methodological issues and clinical applications. Eur Heart J 2006;27:2588-605.

10. Stadler RW, Karl WC, Lees RS. The application of echo-tracking methods to endothelium-dependent vasoreactivity and arterial compliance measurements. Ultrasound Med Biol 1996;22:35-42.

11. Lang RM, Bierig M, Devereux RB, Flachskampf FA, Foster E, Pellikka PA, et al, American Society of Echocardiography's Nomenclature and Standards Committee, Task Force on Chamber Quantification, American College of Cardiology Echocardiography Committee, American Heart Association, European Association of Echocardiography, European Society of Cardiology. Recommendations for chamber quantification. Eur J Echocardiogr 2006;7:79-108.

12. Lancellotti P, Moonen M, Magne J, O'Connor K, Cosyns B, Attena E, et al. Prognostic effect of long-axis left ventricular dysfunction and B-type natri uretic peptide levels in asymptomatic aortic stenosis. Am J Cardiol 2010; 105:383-8.

13. Monin JL, Lancellotti P, Monchi M, Lim P, Weiss E, Piérard L, et al. Risk score for predicting outcome in patients with asymptomatic aortic stenosis. Circulation 2009; 120:69-75.
14. Vriz O, Bossone E, Bettio M, Pavan D, Carerj S, Antonini-Canterin F. Carotid artery stiffness and diastolic function in subjects without known cardiovascular disease. J Am Soc Echocardiogr 2011;24:915-21.

15. Allison MA, Cheung P, Criqui MH, Langer RD, Wright CM. Mitral and aortic annular calcification are highly associated with systemic calcified atherosclerosis. Circulation 2006;113:861-6.

16. Paini A, Boutouyrie P, Calvet D, Tropeano AI, Laloux B, Laurent S. Carotid and aortic stiffness: determinants of discrepancies. Hypertension 2006;47: 371-6.

17. Hansen F, Mangell P, Sonesson B, Länne T. Diameter and compliance in the human common carotid artery-variations with age and sex. Ultrasound Med Biol 1995;21:1-9.

18. Antonini-Canterin F, Roșca M, Beladan CC, Popescu BA, Piazza R, Leiballi $\mathrm{E}$, et al. Echo-tracking assessment of carotid artery stiffness in patients with aortic valve stenosis. Echocardiography 2009;26:823-31.

19. Tobin JR Jr., Rahimtoola SH, Blundell PE, Swan HJ. Percentage of left ventricular stroke work loss. A simple hemodynamic concept for estimation of severity in valvular aortic stenosis. Circulation 1967;35:868-79.

20. Lancellotti P, Donal E, Magne J, O'Connor K, Moonen ML, Cosyns B, et al. Impact of global left ventricular afterload on left ventricular function in asymptomatic severe aortic stenosis: a two-dimensional speckletracking study. Eur J Echocardiogr 2010;11:537-43.

21. Boutouyrie P, Tropeano AI, Asmar R, Gautier I, Benetos A, Lacolley P, et al. Aortic stiffness is an independent predictor of primary coronary events in hypertensive patients: a longitudinal study. Hypertension 2002;39:10-5.

22. Lim P, Monin IL, Monchi M, Garot I, Pasquet A, Hittinger L, et al. Predic tors of outcome in patients with severe aortic stenosis and normal left ventricular function: role of B-type natriuretic peptide. Eur Heart J 2004;25: 2048-53.

23. Levy D, Hwang SJ, Kayalar A, Benjamin EJ, Vasan RS, Parise H, et al. Associations of plasma natriuretic peptide, adrenomedullin, and homocysteine levels with alterations in arterial stiffness: the Framingham Heart Study. Circulation 2007; 115:3079-85.

24. Arnett DK, Chambless LE, Kim H, Evans GW, Riley W. Variability in ultrasonic measurements of arterial stiffness in the Atherosclerosis Risk in Communities study. Ultrasound Med Biol 1999;25:175-80.

25. Liao D, Arnett DK, Tyroler HA, Riley WA, Chambless LE, Szklo M, et al Arterial stiffness and the development of hypertension. The ARIC Study Hypertension 1999;34:201-6. 